\title{
A GENERALIZATION OF THE CYLINDER CONJECTURE FOR DIVISIBLE CODES
}

\author{
SASCHA KURZ, SAM MATTHEUS
}

\begin{abstract}
We extend the original cylinder conjecture on point sets in affine three-dimensional space to the more general framework of divisible linear codes over $\mathbb{F}_{q}$ and their classification. Through a mix of linear programming, combinatorial techniques and computer enumeration, we investigate the structural properties of these codes. In this way, we can prove a reduction theorem for a generalization of the cylinder conjecture, show some instances where it does not hold and prove its validity for small values of $q$. In particular, we correct a flawed proof for the original cylinder conjecture for $q=5$ and present the first proof for $q=7$.

Keywords: cylinder conjecture, linear codes, divisible codes

MSC: Primary 05B25; Secondary 51D20, 51E22.
\end{abstract}

\section{INTRODUCTION}

The cylinder conjecture, as originally formulated in [1], was motivated by direction problems in finite geometry. To be more precise, the strong version of the conjecture is formulated as follows.

Conjecture 1. Let $\mathcal{S}$ be a set of $p^{2}$ points in $\mathrm{AG}(3, p), p$ prime. If $\mathcal{S}$ has the property that every plane is incident with 0 modulo p points of $\mathcal{S}$ then $\mathcal{S}$ is a cylinder, i.e. the union of p parallel lines.

The idea to classify all sets of $p^{2}$ points in $\mathrm{AG}(3, p)$ determining few directions, is a continuation of similar results in $\mathrm{AG}(2, p)$, which started with the work of Rédei and Megyesi [9] and Lovász and Schrijver [8]. The cylinder conjecture as stated above, is of interest from a coding theoretical perspective as well. In particular, one could view this conjecture as part of a more general search for the classification of divisible codes.

A linear $[n, k]_{q}$ code $\mathcal{C}$ is $\Delta$-divisible if all its weights are multiples of a fixed integer $\Delta$. When $\Delta$ and $q$ are coprime, the classification of $\Delta$-divisible codes over $\mathbb{F}_{q}$ is almost trivial, see for example [11] for a general survey on $\Delta$-divisible codes, which contains this result. In this paper, we will focus on the case when $\Delta$ is a power of the field size $q$, hence looking at $q^{r}$-divisible codes, where $r$ is a non-negative integer. For a survey on these codes we refer to [5]. In the case of the original cylinder conjecture, a positive answer would give a classification of all $\left[p^{2}, 4\right]_{p}$ codes that are $p$-divisible as we will explain later on. With the classification of $q^{r}$ divisible codes over $\mathbb{F}_{q}$ in mind it thus makes sense to generalize the cylinder conjecture to higher dimensions and non-prime field characteristic. In order to state and motivate it, we require some terminology and a proper notion of cylinders in higher dimensions. We will therefore defer it to the next section.

\section{PRELIMINARIES}

The notation we will use throughout is the following. Let $V \cong \mathbb{F}_{q}^{v}$ be a $v$-dimensional vector space over the finite field $\mathbb{F}_{q}$ with $q$ elements and $\operatorname{PG}(v-1, q)$ the projective space associated to it. By a $k$-space of $\mathrm{PG}(v-1, q)$ we mean a $k$-dimensional linear subspace of $V$, also using the terms points, lines, planes, and hyperplanes for 1-, 2-, 3-spaces, and $(v-1)$-spaces respectively. A multiset of points in $\mathrm{PG}(v-1, q)$ will be denoted as $\mathcal{M}$, while $\mathcal{S}$ refers to a set of points.

To each multiset $\mathcal{M}$ of $n$ points in $\operatorname{PG}(v-1, q)$ we can assign a $q$-ary linear code $C(\mathcal{M})$ defined by its generator matrix whose $n$ columns consist of representatives of the $n$ points of $\mathcal{M}$. The code $C(\mathcal{M})$ is projective if and only if $\mathcal{M}$ is a set. The weight of a codeword is the number of non-zero coordinates and as mentioned before, a code is called $q^{r}$-divisible if the weight of each codeword is divisible by $q^{r}$.

Definition 2. The multiset $\mathcal{M}$ of $n$ points in $\mathrm{PG}(v-1, q)$ is $q^{r}$-divisible if and only if $C(\mathcal{M})$ is. Equivalently, $\mathcal{M}$ is $q^{r}$-divisible if $|\mathcal{M} \cap H| \equiv|\mathcal{M}|\left(\bmod q^{r}\right)$ for every hyperplane $H$ of $\operatorname{PG}(v-1, q)$. 
For example, the set of points of a $k$-space is $q^{k-1}$-divisible and so is the multiset of the points of a collection of $k$-spaces. The converse gives an interesting question: For which integers $l$ is each $q^{k-1}$ divisible set of $\frac{q^{k}-1}{q-1} \cdot l$ points the union of $l$ (disjoint) $k$-spaces? We can always choose $l \geq 1$ and the maximum value for $l$ implies extendability results of spreads: each set of $q^{k}+1-l$ disjoint $k$-spaces in $\mathbb{F}_{q}^{2 k}$ can be extended to a $k$-spread. These results are mostly formulated in the language of minihypers, see e.g. [4].

An interesting property of $q^{r}$-divisible sets of points is that the divisibility is preserved (to some extent) upon intersecting with subspaces, allowing for inductive arguments.

Lemma 3. ([5, Lemma 7]) Suppose that $\mathcal{M}$ is a $q^{r}$-divisible multiset of $m$ points in $\mathrm{PG}(v-1, q)$ and $X$ a $(v-j)$-space of $\mathrm{PG}(v-1, q)$ with $1 \leq j<r$. Then the restriction $\mathcal{M} \cap X$ is $q^{r-j}$-divisible.

Proof. By induction, it suffices to consider the case $j=1$, i.e. $X=H$ is a hyperplane in $\operatorname{PG}(v-1, q)$.

The hyperplanes of $H$ are the $(v-2)$-subspaces of $\operatorname{PG}(v-1, q)$ contained in $H$. Hence the assertion is equivalent to $|\mathcal{M} \cap U| \equiv|\mathcal{M}|\left(\bmod q^{r-1}\right)$ for every $(v-2)$-subspace $U \subset \mathrm{PG}(v-1, q)$. By assumption we have $\left|\mathcal{M} \cap H_{i}\right| \equiv m\left(\bmod q^{r}\right)$ for the $q+1$ hyperplanes $H_{1}, \ldots, H_{q+1}$ lying above $U$. This gives

$$
(q+1) m \equiv \sum_{i=1}^{q+1}\left|\mathcal{M} \cap H_{i}\right|=q \cdot|\mathcal{M} \cap U|+|\mathcal{M}| \equiv q \cdot|\mathcal{M} \cap U|+m \quad\left(\bmod q^{r}\right)
$$

and hence $m \equiv|\mathcal{M} \cap U|\left(\bmod q^{r-1}\right)$, as claimed.

We can now generalize the concept of a cylinder to higher dimensional vector spaces as follows.

Definition 4. Let $r$ be a non-negative integer. An $(r+1)$-cylinder is a multiset of $q^{r+1}$ points in $\mathrm{PG}(v-1, q)$ that arises as the union of the points of $q$ affine $(r+1)$-subspaces $L_{1} \backslash F, \ldots, L_{q} \backslash F$, where the $L_{i}$ are $(r+1)$-spaces and $F$ is a $r$-space that is contained in all $L_{i}$.

We remark that our definition of a 2-cylinder matches the definition of a cylinder in [3] and the one stated above. By convention a 1-cylinder is just a multiset of $q$ points. As the affine subspaces mentioned in the definition above will appear often, we introduce the notation $A(P, F)$ for the affine subspace $\langle P, F\rangle \backslash F$, where $P$ is a point and $F$ and arbitrary subspace. Note that we have $\operatorname{dim}(A(P, F))=\operatorname{dim}(F)+1$. Next, we observe that $(r+1)$-cylinders can be easily constructed starting from a multiset of $q$ points.

Construction 5. Let $r$ and $v^{\prime}$ be non-negative integers, and consider a $v^{\prime}$-space $V^{\prime}$ and a disjoint $r$-space $F$ in $V=\mathbb{F}_{q}^{v^{\prime}+r}$. If $\mathcal{M}^{\prime}=\left\{P_{1}, \ldots, P_{q}\right\}$ is a multiset of $q$ points in $V^{\prime}$, then a $(r+1)$-cylinder can be constructed as the multiset $\mathcal{M}$ consisting of the points of $A\left(P_{i}, F\right), i=1, \ldots, q$.

Proposition 6. The multiset of points of an $(r+1)$-cylinder is $q^{r}$-divisible.

PROOF. We use the notation of Definition 4 for a given $(r+1)$-cylinder. The statement is trivial for $r=0$ so that we assume $r \geq 1$. Each hyperplane $H$ intersects $F$ either in dimension $r$ or $r-1$. In the first case we have $\left|\left(L_{i} \backslash F\right) \cap H\right| \in\left\{0, q^{r}\right\}$. In the second case we have $\left|\left(L_{i} \backslash F\right) \cap H\right|=q^{r-1}$ for all $1 \leq i \leq q$. Thus, we have $|\mathcal{M} \cap H| \equiv 0\left(\bmod q^{r}\right)$ for the corresponding multiset of points $\mathcal{M}$ of the $(r+1)$-cylinder.

So, $(r+1)$-cylinders yield $q^{r}$-divisible multisets of $q^{r+1}$ points and the question arises if there are other isomorphism types. Indeed there are. Any multiset of $q$ (possibly equal) points with multiplicity $q^{r}$ each is $q^{r}$-divisible. For that reason we will consider sets of points instead of multisets in the remaining part. We remark that studying multisets of points with restricted point multiplicity might be an interesting problem, but we will not go into this here. It will also depend on the dimension whether other isomorphism types exist. Since each set $\mathcal{S}$ of points in $\operatorname{PG}(v-1, q)$ can be embedded in $\mathbb{F}_{q}^{v^{\prime}}$ for $v^{\prime}>v$ we will always assume that $\mathcal{S}$ is spanning, i.e. spans $\mathrm{PG}(v-1, q)$.

Observation 7. In Construction 5, $\mathcal{M}$ is spanning if and only if $\mathcal{M}^{\prime}$ is spanning and $\mathcal{M}$ is a set if and only if $\mathcal{M}^{\prime}$ is a set.

We call an $(r+1)$-cylinder spanning or projective if the corresponding multiset of points is.

Lemma 8. There exists a spanning projective $(r+1)$-cylinder in $\mathrm{PG}(v-1, q)$ if and only if $r+2 \leq v \leq r+q$. 
Proof. Due to the above observations it suffices to remark that $2 \leq \operatorname{dim}\left(\left\langle\mathcal{M}^{\prime}\right\rangle\right) \leq q$ and all dimensions in that range can indeed be attained.

Let $(v, r, q)$ be a triple of non-negative integers where $q$ is a prime power. The question of classification thus boils down to the following.

Question 9. If $\mathcal{S}$ is a $q^{r}$-divisible spanning set of $q^{r+1}$ points in $\mathrm{PG}(v-1, q)$, does it follow that $\mathcal{S}$ must be a $(r+1)$-cylinder?

We say that the generalized cylinder conjecture is true (or false) for a triple $(v, r, q)$ if the answer to this question is affirmative (or not). Notice that the divisibility assumption is as strong as possible and for good reason. It's not hard to construct a $q^{r}$-divisible set of $q^{n}$ points in $\operatorname{PG}(v-1, q), r+1<n \leq v-2$, as the disjoint union of $q^{n-r-1}$ different $(r+1)$-cylinders, while not being a cylinder itself.

As we will see in the next section, it makes no difference if we consider point sets in affine or projective geometries. Therefore, the original cylinder conjecture asserts that the answer is affirmative for the triple $(4,1, p), p$ prime. Remark that when $r=0$, the conjecture is trivially true, so we assume $r \geq 1$ from now on. In Section 3 we will prove some structural results and generalize ideas from [3], using a combination of linear programming and geometrical methods. We discuss some cases where we can determine the validity of the generalized cylinder conjecture in Section 4. Our main results in these two sections can be summarised as follows. In Lemma 3 we have seen that the restriction $\mathcal{S} \cap H$ of a $q^{r}$-divisible set of points $\mathcal{S}$ in $\mathrm{PG}(v-1, q)$ to a hyperplane $H$ is $q^{r-1}$-divisible. We will show that if $|\mathcal{S}|=q^{r+1}$, then there are many hyperplanes $H$ with $|\mathcal{S} \cap H|=q^{r}$, and hence inductive arguments can be applied. Indeed, it will turn out that everything boils down to the case $r=1$, as Corollary 20 shows that the generalized cylinder conjecture is true for $(v, r, q)$ if and only if it is true for $(v-r+1,1, q)$. The generalized cylinder conjecture is not always true: if $q$ is a proper prime power, i.e., not a prime, we can construct counterexamples for suitable dimensions based on the existence of subfields, see Corollary 31. For small values of $q$ we find that the generalized cylinder conjecture is true whenever $q \in\{2,3,5\}$, for the triples $(3,1,4)$ and $(4,1,4)$, but not for $(5,1,4)$. The special case $(4,1, q)$ is treated in detail in Section 5, where the cases $q=5$ (fixing a flawed proof in [3]) and $q=7$ are fully resolved. Although our numerical data is still rather limited, we state:

Conjecture 10. The generalized cylinder conjecture is true for $(r+3, r, q)$ and for $(v, r, p)$ if $p$ is a prime.

Finally, we have to remark that the "right" generalization of the cylinder conjecture to non-prime field sizes is a bit unclear in the context of directions determined by a point set, but Definition 4 and Question 9 are at least reasonable in the context of $q^{r}$-divisible sets.

\section{GENERAL RESUlTS AND THE REDUCTION THEOREM}

The linear programming approach is based on the following three linear equations, commonly referred to as the standard equations.

Lemma 11. (See e.g. [5, Lemma 6])

Let $\mathcal{S}$ be a set of points in $\mathrm{PG}(v-1, q)$ with $|\mathcal{S}|=n$, and let $a_{i}$ be the number of hyperplanes in $\mathrm{PG}(v-1, q)$ containing exactly $i$ points of $\mathcal{S}(0 \leq i \leq n)$. Then we have

$$
\begin{aligned}
\sum_{i=0}^{n} a_{i} & =\frac{q^{v}-1}{q-1}, \\
\sum_{i=1}^{n} i a_{i} & =n \cdot \frac{q^{v-1}-1}{q-1}, \\
\sum_{i=2}^{n}\left(\begin{array}{l}
i \\
2
\end{array}\right) a_{i} & =\left(\begin{array}{l}
n \\
2
\end{array}\right) \cdot \frac{q^{v-2}-1}{q-1} .
\end{aligned}
$$

Proof. Double-count incidences of the tuples $(H),\left(P_{1}, H\right)$, and $\left(\left\{P_{1}, P_{2}\right\}, H\right)$, where $H$ is a hyperplane and $P_{1} \neq P_{2}$ are points contained in $H$. 
The set $\left\{a_{i}\right\}_{i}$ is called the spectrum of $\mathcal{S}$. The set $\mathcal{S}$ being spanning is equivalent to $a_{n}=0$, i.e., no hyperplane contains all points. In that case, the standard equations are equivalent to the first three MacWilliams identities for projective linear codes.

We can adapt Lemma 11 to our situation of $q^{r}$-divisible sets of points.

Lemma 12. Let $\mathcal{S}$ be a $q^{r}$-divisible spanning set of $q^{r+1}$ points in $\mathrm{PG}(v-1, q)$ and let $a_{i q^{r}}$ be the number of hyperplanes in $\mathrm{PG}(v-1, q)$ containing exactly $i q^{r}$ points of $\mathcal{S}(0 \leq i \leq q)$. Then we have

$$
\begin{aligned}
(q-1) \sum_{i=0}^{q-1} a_{i q^{r}} & =q^{v}-1, \\
(q-1) \sum_{i=0}^{q-1} i a_{i q^{r}} & =q\left(q^{v-1}-1\right), \\
(q-1) \sum_{i=0}^{q-1} i\left(i q^{r}-1\right) a_{i q^{r}} & =q\left(q^{r+1}-1\right)\left(q^{v-2}-1\right) .
\end{aligned}
$$

Proof. We use the equations from Lemma 11. Multiplying them by $q-1$, using $n=q^{r+1}$, and taking $q^{r}$-divisibility into account gives

$$
\begin{aligned}
(q-1) \sum_{i=0}^{q} a_{i q^{r}} & =q^{v}-1, \\
(q-1) \sum_{i=0}^{q} i q^{r} a_{i q^{r}} & =q^{r+1}\left(q^{v-1}-1\right), \\
(q-1) \sum_{i=0}^{q}\left(\begin{array}{c}
i q^{r} \\
2
\end{array}\right) a_{i q^{r}} & =\left(\begin{array}{c}
q^{r+1} \\
2
\end{array}\right)\left(q^{v-2}-1\right) .
\end{aligned}
$$

Finally, dividing (8) by $q^{r}$, (9) by $q^{r} / 2$, and recalling $a_{q^{r+1}}=0$ gives the stated result.

Lemma 13. Let $\mathcal{S}$ be a $q^{r}$-divisible spanning set of $q^{r+1}$ points in $\mathrm{PG}(v-1, q)$. Then, the number $a_{q^{r}}$ of hyperplanes with the smallest non-zero number of points is at least $\frac{q^{v}-1}{q-1}-\left(q^{v-r-1}-q+1\right)$.

PROOF. Using the notation from Lemma 12, Equation (6) minus $2 q^{r}-1$ times Equation (5) gives

$$
(q-1) \sum_{i=0}^{q-1} q^{r} i(i-2) a_{i q^{r}}=-q^{r}\left(\left(q^{v}-1\right)-(q-1) q^{v-r-1}+(q-1)^{2}\right) .
$$

Since $i(i-2) \geq 0$ and $a_{i q^{r}} \geq 0$ for all $2 \leq i \leq q-1$, we conclude $(q-1) a_{q^{r}} \geq q^{v}-1-(q-1)\left(q^{v-r-1}-q+1\right)$.

In other words, almost all hyperplanes contain exactly $q^{r}$ points. For these hyperplanes we might apply induction and assume that they are $r$-cylinders, as we will do later on in the proof of Theorem 19.

The general idea behind the proof of Lemma 13 is the linear programming method based on the standard equations, which is a common technique in finite geometry. To be precise, we maximize or minimize a certain $a_{j}$ under the constraints of Lemma 12, where we assume that all $a_{i} \in \mathbb{R}_{\geq 0}$. Bounds similar as in Lemma 13 for other $a_{i q^{r}}$ can be obtained easily.

Lemma 14. Let $\mathcal{S}$ be a $q^{r}$-divisible spanning set of $q^{r+1}$ points in $\mathrm{PG}(v-1, q)$. Then, the number $a_{0}$ of empty hyperplanes is at most $\left(q^{v-r-1}-q+2\right) / 2$.

ProOF. From Lemma 12, $2 q^{r}$ times Equation (4) minus $3 q^{r}-1$ times Equation (5) plus Equation (6) gives

$$
(q-1) \sum_{i=0}^{q-1} q^{r}(i-1)(i-2) a_{i q^{r}}=(q-1)\left(q^{v-1}-q^{r+1}+2 q^{r}\right) .
$$

Since $(i-1)(i-2) \geq 0$ and $a_{i q^{r}} \geq 0$ for all $0 \leq i \leq q-1$, we conclude $2 q^{r} a_{0} \leq\left(q^{v-1}-q^{r+1}+2 q^{r}\right)$. 
Lemma 15. Let $\mathcal{S}$ be a $q^{r}$-divisible spanning set of $q^{r+1}$ points in $\mathrm{PG}(v-1, q)$. Then, the number $a_{0}$ of empty hyperplanes is at least $\frac{q^{v-r-1}-1}{q-1}$.

Proof. Applying Lemma 12, $q^{r}(q-1)$ times Equation (4) minus $q^{r+1}-1$ times Equation (5) plus Equation (6) gives

$$
(q-1) \sum_{i=0}^{q-1} q^{r}(i-1)(i-q+1) a_{i q^{r}}=\left(q^{v-1}-q^{r}\right)(q-1) .
$$

Since $(i-1)(i-q+1) \leq 0$ and $a_{i q^{r}} \geq 0$ for all $1 \leq i \leq q-1$, we conclude $(q-1) a_{0} \geq q^{v-r-1}-1$.

Corollary 16. For every $q^{r}$-divisible spanning set of $q^{r+1}$ points in $\operatorname{PG}(v-1, q)$ there is at least one empty hyperplane.

Proof. Since $\operatorname{PG}(v-1, q)$ contains $\left[\begin{array}{l}v \\ 1\end{array}\right]_{q}=q^{v-1}+q^{v-2}+\cdots+1$ points, we have $v \geq r+2$, so that Lemma 15 gives the stated result.

Thus, it makes no difference if we speak about point sets in $\operatorname{AG}(v-1, q)$ or $\operatorname{PG}(v-1, q)$. Another implication of Corollary 16 is that the generalized cylinder conjecture is trivially true when the dimension $v$ is small.

Proposition 17. Let $\mathcal{S}$ be a $q^{r}$-divisible spanning set of $q^{r+1}$ points in $\mathrm{PG}(v-1, q)$. If $v \leq r+2$, then $v=r+2$ and $\mathcal{S} \simeq \mathrm{AG}(v-1, q)$.

PROOF. As in the proof of Corollary 16 we conclude $v \geq r+2$, so that $v=r+2$. A single empty hyperplane leaves only $q^{r+1}$ possible points, which all have be to contained in $\mathcal{S}$.

We see that the generalized cylinder conjecture is trivially true for all $(v, r, q)$, where $v \leq r+2$. In other words, the classification of $q^{r}$-divisible spanning sets of $q^{r+1}$ points in $\operatorname{PG}(v-1, q)$ is challenging for $v \geq r+3$ only.

With these auxiliary results in hand, we can prove our reduction theorem which essentially states that the validity of the generalized cylinder conjecture depends on the difference $v-r$ and not on the values of $v$ and $r$ itself. As a first step, we show that we can decrease $v$ and $r$ simultaneously and preserve the truthfulness.

Proposition 18. If the generalized cylinder conjecture is true for $(v+1, r+1, q)$, then it is true for $(v, r, q)$.

PROOF. If the generalized cylinder conjecture is false for $(v, r, q)$, we can apply the following construction to the corresponding counterexample and obtain a counterexample for $(v+1, r+1, q)$.

Consider a $q^{r}$-divisible set $\mathcal{S}$ of $q^{r+1}$ points in $V:=\mathrm{PG}(v-1, q)$. For a point $P$ outside of the ambient space we consider the new ambient space $V^{\prime}:=\langle V, P\rangle$ and set $\mathcal{S}^{\prime}=\{A(S, P): S \in \mathcal{S}\}$. By construction $\operatorname{dim}\left(V^{\prime}\right)=v+1$ and $\mathcal{S}^{\prime}$ is a set of $q^{r+2}$ points in $V^{\prime}$. Now let $H^{\prime}$ by a hyperplane of $V^{\prime}$. Either $H^{\prime}=V$ or $H:=H^{\prime} \cap V$ is a hyperplane of $V$. In the first case the have $\mathcal{S}^{\prime} \cap H^{\prime}=\mathcal{S}$, which is of cardinality $q^{r+1}$. In the second case we have $|\mathcal{S} \cap H| \equiv 0\left(\bmod q^{r}\right)$. If $P \leq H^{\prime}$, then $\left|\mathcal{S}^{\prime} \cap H^{\prime}\right|=q \cdot|\mathcal{S} \cap H| \equiv 0\left(\bmod q^{r+1}\right)$. If $P$ is not contained in $H^{\prime}$ then each of the $q^{r+1}$ affine lines $A(S, P)$ is met by $H^{\prime}$ in a single point not equal to $P$, so that $\left|\mathcal{S}^{\prime} \cap H^{\prime}\right|=q^{r+1}$. Thus, $\mathcal{S}^{\prime}$ is $q^{r+1}$-divisible and one can see that it is not a cylinder, if $\mathcal{S}$ is not.

Theorem 19. If the generalized cylinder conjecture is true for $(v, 1, q)$, then it is true for all $(v+r-1, r, q)$.

Proof. Due to Proposition 17 we can assume $v \geq 4$. We will prove the result by induction on $r$. So, assume that the generalized cylinder conjecture is true for $(v+r-2, r-1, q)$. Let $\mathcal{S}$ be a spanning $q^{r}$-divisible set of $q^{r+1}$ points in $\operatorname{PG}\left(v^{\prime}-1, q\right)$, where $v^{\prime}=v+r-1$ and $r \geq 2$. Now let $\mathcal{F}$ be the set of points $F$ such that there exists a point $S \in \mathcal{S}$ with $A(S, F) \subseteq \mathcal{S}$.

We will structure our proof into some intermediate results:

(1) For each point $S \in \mathcal{S}$ there exists an $(r-1)$-space $B$ such that $A(S, B) \subseteq \mathcal{S}$.

(2) $\operatorname{dim}(\langle\mathcal{F}\rangle) \in\{r-1, r\}$.

(3) For each $S_{1}, S_{2} \in \mathcal{S}$ there exists an $(r-1)$-space $B$ such that $A\left(S_{1}, B\right)$ and $A\left(S_{2}, B\right)$ are both contained in $\mathcal{S}$. 
(4) Let $S \in \mathcal{S}$ be a point such that there exist $(r-1)$-spaces $B_{1} \neq B_{2}$ with $A\left(S, B_{1}\right), A\left(S, B_{2}\right) \subseteq \mathcal{S}$. Then, we have $A(S,\langle\mathcal{F}\rangle) \subseteq \mathcal{S}$.

(5) $\mathcal{S}$ is an $(r+1)$-cylinder.

For (1) we use Lemma 13 to conclude that there are at most $q^{v^{\prime}-r-1}-q+1$ hyperplanes that do not contain exactly $q^{r}$ points from $\mathcal{S}$. Thus, for each point $S \in \mathcal{S}$ at least one of the $\frac{q^{v^{\prime}-1}-1}{q-1}$ hyperplanes containing $S$ contains exactly $q^{r}$ points from $\mathcal{S}$. If $H$ is such a hyperplane, we can apply induction for $H$ and conclude that $\mathcal{S} \cap H$ can be partitioned into $\cup_{i=1}^{q} A\left(S_{i}, B\right)$ for some $(r-1)$-space $B$ and $q$ points $S_{i} \in \mathcal{S}$. Of course there exists an index $1 \leq j \leq q$ with $S \in A\left(S_{j}, B\right)$ and hence $A(S, B)=A\left(S_{j}, B\right)$.

For (2) we note that every point $F \in \mathcal{F}$ is contained in every empty hyperplane, as $F$ is the only point in $A(S, F)$ not in $\mathcal{S}$, so that $\langle\mathcal{F}\rangle$ is contained in the intersection of all empty hyperplanes. Since there are exactly $\frac{q^{v^{v^{\prime}}-\operatorname{dim}(\langle\mathcal{F}\rangle)}-1}{q-1}$ hyperplanes containing $\langle\mathcal{F}\rangle$ we conclude from Lemma 15 that $\operatorname{dim}(\langle\mathcal{F}\rangle) \leq r+1$ and moreover if equality holds then every hyperplane containing $\langle\mathcal{F}\rangle$ is empty. Since $\mathcal{S} \neq \emptyset$, this is only possible if $\langle\mathcal{F}\rangle$ is a hyperplane itself, i.e., $v^{\prime}=r+2$ and $v=3$. Thus, we have $\operatorname{dim}(\langle\mathcal{F}\rangle) \leq r$. If $B$ is a subspace according to (1), then $B \subseteq \mathcal{F}$ so that $\operatorname{dim}(\langle\mathcal{F}\rangle) \geq r-1$.

For (3) we can apply the same idea as in (1). Consider the line $L=\left\langle S_{1}, S_{2}\right\rangle$, then $L$ is contained in $\frac{q^{v^{\prime}-2}-1}{q-1}>q^{v^{\prime}-r-1}-q+1$ hyperplanes (using $r \geq 2$ ). So, we can use Lemma 13 to conclude the existence of a hyperplane $H$ with $L \leq H$ and $|\mathcal{S} \cap H|=q^{r}$. Induction on this hyperplane then gives the existence of an $(r-1)$-space $B$ such that $A(S, B) \subseteq \mathcal{S} \cap H$ for all $S \in \mathcal{S} \cap H$.

For (4) we note that $B_{1}, B_{2} \subseteq \mathcal{F}$ implies $\operatorname{dim}(\langle\mathcal{F}\rangle) \geq r$, so that (2) gives $\operatorname{dim}(\langle\mathcal{F}\rangle)=r$.

If $r=2$, then take any point $F$ on the line $\langle\mathcal{F}\rangle$ and a point $S \in \mathcal{S}$. We will directly prove that $A(S, F) \subseteq \mathcal{S}$. We know that there are at most $q^{v^{\prime}-3}-q+1$ hyperplanes not intersecting $\mathcal{S}$ in $q^{2}$ points by Lemma 13, so out of the $q^{v^{\prime}-3}$ hyperplanes through $\langle S, F\rangle$ intersecting $\langle\mathcal{F}\rangle$ in only $F$, there must be at least $q-1$ hyperplanes containing exactly $q^{2}$ points. So take one such hyperplane, apply induction and find that $A(S, F) \subseteq \mathcal{S}$. It follows that $A(S,\langle\mathcal{F}\rangle) \subseteq \mathcal{S}$ for all $S \in \mathcal{S}$, i.e., (4) is valid for $r=2$.

Now assume $r \geq 3$. We can find a point $S \in \mathcal{S}$ and distinct $(r-1)$-spaces $B_{1}, B_{2}$ such that $A\left(S, B_{1}\right)$ and $A\left(S, B_{2}\right)$ both are contained in $\mathcal{S}$. Now, take a point $F \in\langle\mathcal{F}\rangle \backslash\left\{B_{1}, B_{2}\right\}$, and consider any 3-space $\pi$ through $\langle S, F\rangle$ not intersecting $B_{1} \cap B_{2}$. Then this 3-space $\pi$ intersects $B_{1}$ and $B_{2}$ each in a point, say $F_{1}$ and $F_{2}$. There are $\frac{q^{v^{\prime}-3}-1}{q-1}$ hyperplanes through this 3 -space, which is more than $q^{v^{\prime}-r-1}-q+1$ if $r \geq 3$. We again conclude by induction that there must be a $(r-1)$-space $B$ such that $A(S, B) \subseteq \mathcal{S}$. As $F_{1}$ and $F_{2}$ must be contained in $B$, we conclude that $F$ must also be and hence $A(S, F) \subseteq \mathcal{S}$. It again follows that $A(S,\langle\mathcal{F}\rangle) \subseteq \mathcal{S}$ for all $S \in \mathcal{S}$ and so (4) is valid for $r \geq 3$ too.

For the final step (5) we can assume that there exists a point $S \in \mathcal{S}$ such that there is a unique $(r-1)$-space $B$ satisfying $A(S, B) \subseteq \mathcal{S}$, as otherwise by (4) we can already conclude that $\mathcal{S}$ is an $(r+1)$-cylinder. Under this assumption, it follows from (3) that $A\left(S^{\prime}, B\right) \subseteq \mathcal{S}$ for all $S^{\prime} \in \mathcal{S}$, so that modulo $B$ we obtain a set $\mathcal{S}^{\prime}$ of $q^{2}$ points that is $q$-divisible and spans a space of dimension $v^{\prime}-r+1=v$. For this set $\mathcal{S}^{\prime}$ we can apply the generalized cylinder conjecture for $(v, 1, q)$ and conclude $\mathcal{S}^{\prime}=\cup_{i=1}^{q} A\left(S_{i}^{\prime}, B^{\prime}\right)$ for some points $S_{i}^{\prime}$ and $B^{\prime}$. By construction, we then have $\mathcal{S}=\cup_{i=1}^{q} A\left(S_{i}^{\prime},\left\langle B, B^{\prime}\right\rangle\right)$ which shows that $\mathcal{S}$ is an $(r+1)$-cylinder.

Combining the previous results, we find the promised reduction theorem.

Corollary 20. The generalized cylinder conjecture is true for $(v, r, q)$ if and only if it is true for $(v-r+1,1, q)$.

Finally, we can transfer many of the insights of [3] to our more general situation.

Lemma 21. (Cf. [3, Lemma 1])

Let $\mathcal{S}$ be a $q^{r}$-divisible spanning set of $q^{r+1}$ points in $\mathrm{PG}(v-1, q)$. Let $K$ be a subspace of codimension 2 in $\mathrm{PG}(v-1, q)$. Assume that $|\mathcal{S} \cap K|=k q^{r-1}$ for some integer $0<k<q$. Then, any hyperplane containing $K$ contains at most $k q^{r}$ points from $\mathcal{S}$.

PROOF. Since every hyperplane containing $K$ contains at least $k q^{r-1}$ points, it should contain at least $q^{r}$ points. Therefore, counting the number of points on hyperplanes containing $K$, we find at least $(q+$ 
1) $\left(q^{r}-k q^{r-1}\right)+k q^{r-1}=q^{r+1}-(k-1) q^{r}$ points. Hence, there are $(k-1) q^{r}$ points left, which implies that a single hyperplane contains at most $k q^{r}$ points.

Corollary 22. (Cf. [3, Corollary 1])

Let $\mathcal{S}$ be a $q^{r}$-divisible spanning set of $q^{r+1}$ points in $\mathrm{PG}(v-1, q)$. Suppose the hyperplane $H$ contains $k q^{r}$ points of $\mathcal{S}$, where $0<k<q$, then every hyperplane $K$ of $H$, i.e., $K \leq H$ is a subspace of codimension 2 in $\mathrm{PG}(v-1, q)$, contains either 0 or $l q^{r-1}$ points of $\mathcal{S}$ for some $k \leq l \leq q$.

Proof. By Lemma 3 we know that $|\mathcal{S} \cap K| \equiv 0\left(\bmod q^{r-1}\right)$ holds . Suppose that $K$ contains $l q^{r-1}$ points for some integer $0<l<k$. Then, by Lemma 21, every hyperplane $H$ in $\mathrm{PG}(v-1, q)$ containing $K$ contains at most $l q^{r}<k q^{r}$ points, which is a contradiction.

For the next proof, denote by $[x]_{q}:=\left(q^{x}-1\right) /(q-1)$ the number of hyperplanes through a codimension $x$-space.

Theorem 23. (Cf. [3, Theorem 2])

Let $\mathcal{S}$ be a $q^{r}$-divisible spanning set of $q^{r+1}$ points in $\mathrm{PG}(v-1, q)$. If $\mathcal{S}$ contains a full affine $(r+1)$-space then $\mathcal{S}$ is a $(r+1)$-cylinder.

Proof. Due to Proposition 17 we can assume $v \geq r+3$. Denote by $A$ the affine $(r+1)$-space contained in $\mathcal{S}$ and let $A_{\infty}$ be 'its part at infinity', i.e. the unique $r$-space so that $A \cup A_{\infty}$ is an $(r+1)$-space of $\mathrm{PG}(v-1, q)$. Take a point $P \in \mathcal{S} \backslash A$ and let $H$ be a hyperplane containing $A$ and $P$. By $q^{r}$-divisibility, we can assume that $|\mathcal{S} \cap H|=k q^{r}$ with $1<k<q$.

Fix a point $Q \in A$ and consider the set $\mathcal{K}$ of $(v-2)$-spaces through $Q$, not containing $A_{\infty}$. Observe that $|\mathcal{K}|=[v-2]_{q}-[v-r-2]_{q}$ and that every point in $(\mathcal{S} \cap H) \backslash A$ is contained in $a:=[v-3]_{q}-[v-r-3]_{q}$ of these spaces. Now double counting pairs $\{(K, R) \mid K \in \mathcal{K}, R \in K \cap \mathcal{S} \backslash A\}$ we find

$$
\left(k q^{r}-q^{r}\right) a=\sum_{K \in \mathcal{K}}|(K \cap \mathcal{S} \backslash A)| \geq|\mathcal{K}|\left(k q^{r-1}-q^{r-1}\right),
$$

by Corollary 22. As $|\mathcal{K}|=a q$, we see that in fact we have equality so that $|K \cap \mathcal{S}|=k q^{r-1}$ for all $K \in \mathcal{K}$. Moreover, the point $Q \in A$ was arbitrary so we conclude that every $(v-2)$-space not through $A_{\infty}$ contains $k q^{r-1}$ points.

Now retaking the point $P \in \mathcal{S} \cap H \backslash A$, we can consider the set $\mathcal{K}^{\prime}$ of $(v-2)$-spaces through $P$, not containing $A_{\infty}$. Again, this set has size $\left|\mathcal{K}^{\prime}\right|=[v-2]_{q}-[v-r-2]_{q}$. Denote by $B$ the $(r+1)$-space $\left\langle A_{\infty}, P\right\rangle$ and $x=|\mathcal{S} \cap B|$. If we can show that $x=q^{r}$, we are done. We can now double count the pairs $\left\{(K, R) \mid K \in \mathcal{K}^{\prime}, R \in K \cap \mathcal{S} \backslash\{P\}\right\}$. If $R \in B$ then the number of $(v-2)$-spaces through $P$ and $R$, not containing $A_{\infty}$ is $b=[v-3]_{q}-[v-r-2]_{q}$. For a point not in $B$, the correct count for the $(v-2)$-spaces is still $a$ and we find the following equality:

$$
\left(k q^{r}-x\right) a+(x-1) b=\sum_{K \in \mathcal{K}^{\prime}}|(K \cap \mathcal{S} \backslash B)|=\left|\mathcal{K}^{\prime}\right|\left(k q^{r-1}-1\right) .
$$

After simplification we indeed find $x=q^{r}$, concluding the proof.

\section{Positive AND REgative RESUlts}

In this section we will discuss some triples $(v, r, q)$ for which we can determine the validity of the generalized cylinder conjecture. Before that, we introduce some notation to make the proofs less cumbersome.

If $K$ is a $k$-space in $\mathrm{PG}(v-1, q)$, then $K(\mathcal{S}):=|K \cap \mathcal{S}|$ denotes the number of points in the intersection, which we will also refer to as the multiplicty of $K$. If $K$ is a point, then we will say that $K$ is a 0 - or 1-point, whenever $K(\mathcal{S})=0$ or 1 respectively. Similarly, if $K$ is a line or a plane, we will say that $K$ is an $m$-line or $m$-plane if $K(\mathcal{S})=m$.

First of, we will show that the generalized cylinder conjecture is true whenever $q \in\{2,3\}$.

Lemma 24. Let $\mathcal{S}$ be a spanning set of $q^{r+1}$ points in $\mathrm{PG}(v-1, q)$. If every hyperplane of $\mathrm{PG}(v-1, q)$ contains either $q^{r}$ or no point from $\mathcal{S}$, then $v=r+2$ and $\mathcal{S} \simeq \operatorname{AG}(v-1, q)$. 
PROOF. Since only $a_{0}$ and $a_{q^{r}}$ are non-zero in Lemma 12, we immediately find $v=r+2$, so that we can apply Proposition 17.

Corollary 25. The generalized cylinder conjecture is true for all triples $(v, r, 2)$.

Next up, we will show the generalized cylinder conjecture for $(v, 1,3)$. By Corollary 20, this suffices to conclude that the generalized cylinder conjecture is true for all triples $(v, r, 3)$.

Proposition 26. Let $\mathcal{S}$ be a 3-divisible spanning set of 9 points in $\mathrm{PG}(v-1,3)$. Then $\mathcal{S}$ is a 2-cylinder.

Proof. Assume to the contrary, that $\mathcal{S}$ is not a 2-cylinder, so that Proposition 17 implies $v \geq 4$. Since the maximum multiplicity of a hyperplane is 6 , each subspace of multiplicity 6 is a hyperplane. Assume that $K$ is a subspace of multiplicity 4 , so that $\operatorname{dim}(K) \leq v-2$. We denote the codimension $v-\operatorname{dim}(K)$ of $K$ by $x$, and hence $x \geq 2$. Since there are $[x]_{3}$ hyperplanes through $K$, every 1-point outside of $K$ is contained in $[x-1]_{3}$ hyperplanes, and every hyperplane through $K$ has multiplicity 6 , we have $2[x]_{3}=5[x-1]_{3}$, so that $3^{x-1}=-3$, which is impossible. Thus, no subspace can have a multiplicity of exactly 4 .

By Lemma 24 we can assume the existence of a hyperplane $H$ with $\mathcal{S}(H)=6$. Since $\mathcal{S}$ is spanning we have $\operatorname{dim}(H) \leq 6$. If $\operatorname{dim}(H)=6$, then we can assume w.l.o.g. that the 1-points in $H$ are given by $\left\langle e_{1}\right\rangle, \ldots,\left\langle e_{6}\right\rangle$, where the $e_{i}$ denote the standard unit vectors. With this, the subspace $\left\langle e_{1}, \ldots, e_{4}\right\rangle$ would have multiplicity 4 , which is a contradiction. Now assume $\operatorname{dim}(H)=5$ and that the 1-points in $H$ are given by $\left\langle e_{1}\right\rangle, \ldots,\left\langle e_{5}\right\rangle$ and a sixth point $P$. Consider the subspace $\left\langle e_{1}, \ldots, e_{4}\right\rangle$. Since it does not contain $e_{5}$ and there is no subspace of multiplicity four, it has to contain $P$, which means that the fifth coordinate of the vectors in $P$ are zero. We can repeat this argument for the subspace $\left\langle\left\{e_{1}, \ldots, e_{5}\right\} \backslash e_{i}\right\rangle, i=1, \ldots, 4$ and conclude that the $i$-th coordinate of the vectors in $P$ are zero for all $1 \leq i \leq 5$, which is a contradiction. Thus, the remaining possibilities are $\operatorname{dim}(H) \in\{3,4\}$, i.e., $v \in\{4,5\}$. From Theorem 23 we conclude that the maximum line multiplicity is at most 2. So, if $\operatorname{dim}(H)=3$, then $\mathcal{S} \cap H$ would be a set of 6 points in $\mathrm{PG}(2,3)$ with line multiplicity at most two, which does not exist. Therefore, we have $\operatorname{dim}(H)=4$ and $v=5$. Using Corollary 22 we conclude that every plane $\pi$ in $H$ has a multiplicity in $\{0,2,3,5\}$. By the same reasoning as before, we cannot have 5 points in $\mathrm{PG}(2,3)$ with line multiplicity at most two and hence $\mathcal{S}(\pi) \neq 5$. For the spectrum $\left(a_{i}^{\prime}\right)$ of $\mathcal{S} \cap H$ the standard equations yield the unique solution $a_{0}^{\prime}=8, a_{2}^{\prime}=18, a_{3}^{\prime}=14$. Now consider the subspaces spanned by one of the $\left(\begin{array}{l}6 \\ 3\end{array}\right)=20$ triples of 1-points in $H$. As no line contains 3 points and every plane contains at most 3 points, each triple of points spans a distinct plane, implying $a_{3}^{\prime} \geq 20$, which is a contradiction.

Corollary 27. The generalized cylinder conjecture is true for all triples $(v, r, 3)$.

An interesting implication is that the dimension $v$ of every $3^{r}$-divisible projective $\left[3^{r+1}, v\right]_{3}$-code is at most $r+3$ for every positive integer $r$. We remark that Ward's upper bound on the dimension of divisible codes [11, Theorem 6] gives $v \leq q(r+1)$ for the triple $(v, r, q)$, and is hence not strong enough to give this result. Using the software package LinCode [6] we have computationally checked that there is no 3-divisible $[9, \geq 5]_{3}$-code, no 9 -divisible $[27, \geq 6]_{3}$-code, and no 27 -divisible $[81, \geq 7]_{3}$-code. This means it might not be necessary to assume that $\mathcal{S}$ is a set and not a multiset to obtain the stated upper bound for the dimension. We remark that the truth of the cylinder conjecture for $(4,1,2)$ and $(4,1,3)$ was also proven in [3].

In principle it is possible to enumerate all projective $q^{r}$-divisible $\left[q^{r+1}, v\right]_{q}$ codes and to check whether the corresponding point sets are $(r+1)$-cylinders for given finite parameters. However, given the currently available software for the exhaustive enumeration of linear codes, this approach is limited to rather small parameters. Nevertheless we report our corresponding findings here. The last step - checking whether all resulting point sets are $(r+1)$-cylinders - can be replaced by a counting argument. The numbers of projective linear codes over $\mathbb{F}_{5}$ of effective lengths $n=5$ ordered by their dimension $k$ are given by $2^{1} 3^{4} 4^{3} 5^{1}$, as can be easily enumerated using the software package LinCode [6] - even a classification by hand is possible. So, Construction 5 yields $3^{1} 4^{4} 5^{3} 6^{1} 5$-divisible projective linear codes over $\mathbb{F}_{5}$ of effective lengths $n=25$, again ordered by their dimension $k$. Using LinCode we verified that there are no further 5 -divisible projective linear codes over $\mathbb{F}_{5}$ of effective length $n=25$. Thus, we have computationally proven that the generalized cylinder conjecture is true for $(v, 1,5)$, where the dimension $v$ is arbitrary. This covers the special case $(4,1,5)$ that we treat in the subsequent section. From Corollary 20 we conclude: 
Corollary 28. The generalized cylinder conjecture is true for all triples $(v, r, 5)$.

For $q \in\{2,3,4\}$ we can perform the same computation. The cases $q=2,3$ verify our theoretical findings for $(v, 1, q)$. The number of projective linear codes over $\mathbb{F}_{4}$ of effective lengths $n=4$ ordered by their dimension $k$ are given by $2^{1} 3^{2} 4^{1}$. The number of 4 -divisible projective linear codes over $\mathbb{F}_{4}$ of effective lengths $n=16$ ordered by their dimension $k$ are given by $3^{1} 4^{2} 5^{2}$. In other words, the generalized cylinder conjecture is true for $(3,1,4)$ and $(4,1,4)$ but not for $(5,1,4)$. For $v \geq 6$ there do not exist projective 4-divisible $[16, v]_{4}$-codes so that the generalized cylinder conjecture is trivially true for $(v, 1,4)$ whenever $v \geq 6$. Therefore, Corollary 20 gives:

Corollary 29. The generalized cylinder conjecture is true for $(v, r, 4)$ if and only if $v \neq r+4$.

Of the two linear codes in dimension 5 , the one that does not correspond to a 2 -cylinder has a generator matrix given by

$$
\left(\begin{array}{l}
0110101101110000 \\
1101100011101000 \\
1100011111000100 \\
1111111000000010 \\
0111010110100001
\end{array}\right) .
$$

The code has weight enumerator $W(z)=1 z^{0}+90 z^{8}+840 z^{12}+93 z^{16}$ and an automorphism group of order 1935360. Considered over $\mathbb{F}_{2}$ the stated generator matrix gives a linear $\mathbb{F}_{2}$ code with weight enumerator $W(z)=1 z^{0}+30 z^{8}+1 z^{16}$, which means that the code is an affine 5 -space.

Computationally we also verified that the generalized cylinder conjecture is true for $(5,2,4)$, which also follows from Theorem 19. Due to the counter example for $(5,1,4)$ there is also a counter example for $(6,2,4)$, see Proposition 18 . We have computationally checked that this counter example is unique.

In order to generalize the above counter example to the generalized cylinder conjecture for $(5,1,4)$ we remark that for each integer $h \geq 2$ the field $\mathbb{F}_{q}$ is a subfield of $\mathbb{F}_{q^{h}}$, so that $\mathbb{F}_{q^{h}}^{v} \cong \mathbb{F}_{q}^{v h}$. Using this isomorphism we can we can embed every multiset of points $\mathcal{M}^{\prime}$ in $\mathbb{F}_{q}^{v}$ as a multiset of points $\mathcal{M}$ (of the same cardinality) in $\mathbb{F}_{q^{h}}^{v}$. Moreover, every $k$-space in $\mathbb{F}_{q^{h}}^{v}$ corresponds to a $k h$-space in $\mathbb{F}_{q}^{v h}$.

Lemma 30. Let $\mathcal{S}^{\prime}$ be a spanning projective $2 h$-cylinder in $\mathbb{F}_{q}^{v}$, where $h \geq 2$. Then the corresponding embedding $\mathcal{S}$ in $\mathbb{F}_{q^{h}}^{v} \cong \mathbb{F}_{q}^{v h}$ is a spanning projective $q^{h}$-divisible set of $\left(q^{h}\right)^{2}$ points in $\mathrm{PG}\left(v-1, q^{h}\right)$ that is not a 2-cylinder

Proof. First we observe $|\mathcal{S}|=\left|\mathcal{S}^{\prime}\right|=q^{2 h}=\left(q^{h}\right)^{2}$. Since $\mathcal{S}^{\prime}$ is spanning and projective, the same applies to $\mathcal{S}$. An arbitrary hyperplane $H$ in $\mathbb{F}_{q^{h}}^{v}$ has dimension $(v-1) h$ over $\mathbb{F}_{q}$. Since $\mathbb{F}_{q}^{v}$ has dimension $v$ over $\mathbb{F}_{q}$, there exists a subspace $K$ in $\mathbb{F}_{q}^{v}$ of dimension at least $v-h$ such that $|\mathcal{S} \cap H|=\left|\mathcal{S}^{\prime} \cap K\right|$. Note that $\mathcal{S}^{\prime}$ is $q^{2 h-1}$-divisible, so that $\left|\mathcal{S}^{\prime} \cap K\right| \equiv 0\left(\bmod q^{h}\right)$ due to Lemma 3. and we conclude that $\mathcal{S}$ is $q^{h}$-divisible.

Now assume that $\mathcal{S}$ is a 2-cylinder and let $L$ be one of the $q^{h}$-lines. Consider two 1-points $P_{1}, P_{2}$ on $L$ and denote by $P_{1}^{\prime}, P_{2}^{\prime}$ be the corresponding points in $\mathcal{S}^{\prime}$. The line $L^{\prime}=\left\langle P_{1}^{\prime}, P_{2}^{\prime}\right\rangle$ has multiplicity at most $q$ in $\mathcal{S}^{\prime}$, so that $L$ has a multiplicity of at most $q$ in $\mathcal{S}$, which is a contradiction due to $h \geq 2$.

Corollary 31. For any integer $h \geq 2$, the generalized cylinder conjecture is false for $\left(v, r, q^{h}\right)$ whenever $2 h+r \leq v \leq 2 h+r+q-2$.

PROOF. From Lemma 30 and Lemma 8 we conclude that the generalized cylinder conjecture is wrong for $\left(v, 1, q^{h}\right)$, where $2 h+1 \leq v \leq 2 h-1+q$, so that Corollary 20 gives the general statement.

If $v=2 h+r$, then the point set $\mathcal{S}^{\prime}$ in Lemma 30 is an affine geometry, so that $\mathcal{S}$ is an affine subgeometry. For the special case $h=2$ one also speaks of a Baer (sub-)geometry. In general, our construction is an instance of the technique of the so-called field reduction, which yields a lot of non-trivial constructions and characterizations of geometric and algebraic structures, see e.g. [7]. Of course one might conjecture that every $q^{r}$-divisible set of $q^{r+1}$ points in $\mathrm{PG}(v-1, q)$ is either an $(r+1)$-cylinder or arises from a cylinder over a subfield. 


\section{The GenerAlized CYLINDER CONJECTURE FOR $(4,1, q)$}

In this section, we will focus on the case $v=r+3$, and by Corollary 20 we can restrict ourselves to the triple $(4,1, q)$. We will gather some more information on a possible counterexample, which leads us to be able to prove the generalized cylinder conjecture for $(4,1,5)$ and $(4,1,7)$. A proof of the former was also claimed in [3], but the proof contains an error which we correct here.

For the results in this section, we will often make the following assumption:

$(\star) \quad \mathcal{S}$ is a $q$-divisible spanning set of $q^{2}$ points in $\mathrm{PG}(3, q)$ which is not a 2-cylinder.

Since $r$ is fixed in this section, we will also refer to a 2-cylinder as a cylinder. Moreover, we can consider $\mathcal{S}$ as a set of points in $\operatorname{AG}(3, q)$ by Corollary 16. Lastly, by Theorem 23 we can assume that any plane intersects $\mathcal{S}$ in at most $q^{2}-q$ points. Our general strategy is to obtain some structural results on $\mathcal{S}$ and find a contradiction for small $q$. We will heavily rely on the standard equations for planes and points in $\mathcal{S}$ as stated in Lemma 12, but also similar equations for lines in a $k q$-plane $H$ and points in $\mathcal{S} \cap H$, obtained by the same double counting method. The latter gives us information on the number of $i$-lines for each $i$, which we will also refer to as the spectrum.

We start off by investigating the multiplicity of a line with respect to $\mathcal{S}$. Summarizing the conclusions of Corollary 16, Corollary 22 and Theorem 23, we can state the following lemma.

Lemma 32. Under $(\star)$, the line multiplicities in a $k q$-plane $H$ are contained in $\{0, k, k+1, \ldots, q-1\}$.

The restriction on the possible line multiplicities is quite severe. Indeed, we can investigate the existence of a set $\mathcal{K}$ of $k q$ points in $\operatorname{PG}(2, q)$ admitting these line multiplicities, independently of the (generalized) cylinder conjecture. It turns out that when $k$ is large, such sets cannot exist and hence $\mathcal{S}$ cannot have large intersections with planes. This idea is illustrated in the next few results.

Lemma 33. For a set of $q(q-1)$ points in $\mathrm{PG}(2, q)$, there exists a line with a multiplicity not in $\{0, q-1\}$.

Proof. Otherwise the first two standard equations would give $a_{0}=1$ and $a_{q-1}=q^{2}+q$, so that the third one yields the contradiction

$$
\left(\begin{array}{c}
q-1 \\
2
\end{array}\right) a_{q-1}=\frac{q(q-1)\left(q^{2}-q-2\right)}{2}<\frac{q(q-1)\left(q^{2}-q-1\right)}{2}=\left(\begin{array}{c}
q(q-1) \\
2
\end{array}\right) .
$$

Corollary 34. Under $(\star)$, there can be no $q(q-1)$-plane.

ProOF. If $H$ is a $q(q-1)$-plane, then Lemma 32 and Lemma 33 yield a contradiction.

We can use this result to find an alternative proof of the cylinder conjecture when $q \in\{2,3\}$.

Corollary 35. The generalized cylinder conjecture is true for the triples $(4,1,2)$ and $(4,1,3)$.

Proof. Assume that $\mathcal{S}$ is not a cylinder, so that Corollary 34 implies $a_{q(q-1)}=0$. For $q=2$ this means that all points of $\mathcal{S}$ are contained in 0 -planes, which is absurd. For $q=3$ solving the first two standard equations give $a_{0}=1$ and $a_{3}=39$. The third implies the contradiction

$$
\left(\begin{array}{l}
3 \\
2
\end{array}\right) a_{3}=117<144=\left(\begin{array}{l}
9 \\
2
\end{array}\right) \cdot(3+1) .
$$

Remark 36. The argumentation of the proof of Corollary 35, i.e., the first three standard equations combined with Corollary 34, would give the unique spectrum $a_{0}=7, a_{1}=72$, and $a_{2}=6$ for $q=4$.

Lemma 37. For a set of $q(q-2)$ points in $\mathrm{PG}(2, q), q \geq 4$, there exists a line with a multiplicity not in $\{0, q-2, q-1\}$. 
PROOF. Otherwise the first two standard equations would give $a_{q-1}=a_{0}(q-2)-(q-2)$ and $a_{q-2}=$ $q^{2}+2 q-1-a_{0}(q-1)$, so that the third yields

$$
0=a_{q-2}\left(\begin{array}{c}
q-2 \\
2
\end{array}\right)+a_{q-1}\left(\begin{array}{c}
q-1 \\
2
\end{array}\right)-\left(\begin{array}{c}
q(q-2) \\
2
\end{array}\right)=\frac{(q-2)\left(a_{0}(q-1)-3 q+1\right)}{2}
$$

which implies

$$
a_{0}=\frac{3 q-1}{q-1}=3+\frac{2}{q-1} \notin \mathbb{N}_{0} .
$$

Remark 38. In the proof of Lemma 37 the third standard equation is needed since e.g. $\left(a_{0}, a_{q-2}, a_{q-1}\right)=$ $(2,17,2)$ satisfies the first two standard equations for $q=4$.

Corollary 39. Under $(\star)$, there can be no $q(q-2)$-plane.

Proof. Due to Corollary 35 we can assume $q \geq 4$. If $H$ is a $(q-2) q$-plane, then Lemma 32 and Lemma 37 yield a contradiction.

Corollary 40. The generalized cylinder conjecture is true for the triple $(4,1,4)$.

Proof. Assume that $\mathcal{S}$ is not a cylinder, so that Corollary 34 and Corollary 39 imply $a_{q(q-1)}=0$ and $a_{q(q-2)}=0$. For $q=4$ solving the first two standard equations give $a_{0}=1$ and $a_{4}=84$. The third implies the contradiction

$$
\left(\begin{array}{l}
4 \\
2
\end{array}\right) a_{4}=504<600=\left(\begin{array}{c}
16 \\
2
\end{array}\right) \cdot(4+1) .
$$

We might continue in the vein of Lemma 37 and consider sets of $q(q-3)$ points in $\mathrm{PG}(2, q)$ whose line multiplicities are contained in $\{0, q-3, q-2, q-1\}$. Due to to Corollary 35 and Corollary 40 we are only interested in the cases where $q \geq 5$. It turns out that he unique possibility is given by $q=5$ and a spectrum given by $a_{0}=6, a_{2}=15, a_{3}=10$, and $a_{4}=0$. So, similar to Corollary 39, we can conclude that a $q(q-3)$-plane does not exist unless $q=5$. This result also follows in a much shorter way from Lemma 43, but we want to remark that the latter is not necessary to show that the generalized cylinder conjecture is true for the triple $(4,1,5)$.

Proposition 41. The generalized cylinder conjecture is true for the triple $(4,1,5)$.

Proof. By Corollary 34 and Corollary 39 we know that $a_{q(q-1)}=0$ and $a_{q(q-2)}=0$. With this, the standard equations for $\mathcal{S}$ yield the unique spectrum $a_{0}=11, a_{5}=135$, and $a_{10}=10$. Now let $H$ be a 10 -plane and $b_{i}$ the number of lines of multiplicity $i$, where $b_{i}=0$ for $i \notin\{0,2,3,4\}$. From the standard equations we conclude $b_{2}=51-6 b_{0}, b_{3}=-38+8 b_{0}$, and $b_{4}=18-3 b_{0}$, so that $b_{4} \geq 0$ implies $b_{0} \leq 6$ and $b_{3} \geq 0$, $b_{0} \in \mathbb{N}_{0}$ imply $b_{0} \geq\left[\frac{19}{4}\right]=5$. Assume $b_{0}=5$ and note that two 4-lines cannot share a common 1 -point $P$ since otherwise counting points on the lines $L_{1}, \ldots, L_{6}$ through $P$ would yield the contradiction

$$
10=\mathcal{S}(H)=\sum_{i=1}^{6} \mathcal{S}\left(L_{i}\right)-5 \cdot \mathcal{S}(P) \geq 2 \cdot 4+4 \cdot 2-5=11
$$

using $\mathcal{S}\left(L_{i}\right) \geq 2$ for all $1 \leq i \leq 6$. Thus, the 4 -lines are pairwise disjoint, so that $10=\mathcal{S}(H) \geq a_{4} \cdot 4=12$, which is a contradiction. Hence, we have $b_{0}=6, b_{2}=15, b_{3}=10$, and $b_{i}=0$ otherwise.

For a line $L$ of multiplicity 3 in $H$ denote the other 5 planes by $H_{1}, \ldots, H_{5}$. Since $\mathcal{S}\left(H_{i}\right) \in\{5,10\}$ for all $1 \leq i \leq 5$ and $|\mathcal{S}|=25$, there exists an index $1 \leq i \leq 5$ with $\mathcal{S}\left(H_{i}\right)=10$. Due to $1+b_{3} \cdot 1=11$, there are at least eleven 10-planes, which contradicts $a_{10}=10$.

Remark 42. There exists a unique projective $[10,3,\{6,7,8,10\}]_{5}$ code $\mathcal{C}$ with generator matrix

$$
\left(\begin{array}{llllllllll}
1 & 1 & 1 & 1 & 1 & 1 & 0 & 1 & 0 & 0 \\
4 & 4 & 3 & 2 & 1 & 0 & 1 & 0 & 1 & 0 \\
3 & 4 & 4 & 2 & 0 & 1 & 4 & 0 & 0 & 1
\end{array}\right)
$$


This code has an automorphism group of order 480 and weight enumerator $W_{\mathcal{C}}(x)=1 x^{0}+40 x^{7}+60 x^{8}+$ $24 x^{10}$. A geometrical interpretation for this set of points was also given in [10]. We remark that the existence of the above code was excluded in the proof of [3, Theorem 4] and so the proof is flawed. More precisely, in the last sentence of the argument showing the existence of a 4-line in a 10-plane where two further 0-lines are constructed, it can happen that they (partially) coincide with the four 0-lines found before.

Lemma 43. Under $(\star)$, the maximum line multiplicity with respect to $\mathcal{S}$ is $q-2$.

Proof. Due to Corollary 35 and Corollary 40 we can assume $q \geq 5$. By Lemma 32 we already know that the maximum line multiplicity is at most $q-1$. So assume that $L$ is a line of multiplicity $q-1$ and denote by $Q_{1}, Q_{2}$ the two 0 -points on $L$. Let $H$ be an arbitrary hyperplane containing $L$, where $\mathcal{S}(H)=k q$ for an integer $1 \leq k<q$.

For each 1-point $P$ on $L$ let $L_{1}, \ldots, L_{q}$ denote the $q$ lines trough $P$ in $H$ that are not equal to $L$. Note that Lemma 32 implies $\mathcal{S}\left(L_{i}\right) \geq k$ for all $1 \leq i \leq q$. From

$$
k q=\mathcal{S}(H)=\mathcal{S}(L)+\sum_{i=1}^{k} \mathcal{S}\left(L_{i}\right)-q \cdot \mathcal{S}(P) \geq q-1+q k-q=k q-1
$$

we conclude that $q-1$ of the $L_{i}$, where $1 \leq i \leq q$, have multiplicity $k$ and one has multiplicity $k+1$.

Now consider a 0-point $R$ in $H$ not on $L$ and let $L_{i}^{\prime}$ be the lines through $R$ and $Q_{i}$ in $H$, where $1 \leq i \leq 2$. By $L_{3}^{\prime}, \ldots, L_{q+1}^{\prime}$ we denote the remaining $q-1$ lines through $R$ in $H$. Note that the $L_{i}^{\prime}$ meet the line $L$ in a 1-points, so that $\mathcal{S}\left(L_{i}^{\prime}\right) \in\{k, k+1\}$ for $3 \leq i \leq q+1$. From

$$
k q=\mathcal{S}(H)=\sum_{i=1}^{q+1} \mathcal{S}\left(L_{i}^{\prime}\right)-q \cdot \mathcal{S}(R) \geq \mathcal{S}\left(L_{1}^{\prime}\right)+\mathcal{S}\left(L_{2}^{\prime}\right)+(q-1) k
$$

we conclude $\mathcal{S}\left(L_{1}^{\prime}\right)+\mathcal{S}\left(L_{2}^{\prime}\right) \leq k$, so that $\mathcal{S}\left(L_{1}^{\prime}\right), \mathcal{S}\left(L_{2}^{\prime}\right) \in\{0, k\}$ due to Lemma 32. Thus, for $1 \leq i \leq 2$ the $q+1$ lines through $Q_{i}$ in $H$ are given by the $(q-1)$-line $L, \frac{q-1}{k}$ lines of multiplicity 0 , and $\left(q-\frac{q-1}{k}\right)$ lines of multiplicity $k$. Now we are ready to determine the spectrum $\left(a_{i}\right)$ of $H$ :

$$
\begin{aligned}
a_{0} & =2 \cdot \frac{q-1}{k} \\
a_{k} & =(q-1) \cdot(q-1)+2 \cdot\left(q-\frac{q-1}{k}\right) \\
a_{k+1} & =(q-1) \cdot 1 \\
a_{q-1} & =1
\end{aligned}
$$

and $a_{i}=0$ for all $i \notin\{0, k, k+1, q-1\}$. If $k=q-1$ or $k+1=q-1$, then we would have to take the sum of both values, but we suppress this technical subtlety for the ease of notation. From the third standard equation we conclude

$$
0=\left(\begin{array}{l}
k \\
2
\end{array}\right) a_{k}+\left(\begin{array}{c}
k+1 \\
2
\end{array}\right) a_{k+1}+\left(\begin{array}{c}
q-1 \\
2
\end{array}\right) a_{q-1}-\left(\begin{array}{c}
k q \\
2
\end{array}\right)=q \cdot \frac{(k-1)(k-q+1)}{2},
$$

so that $k \in\{1, q-1\}$.

So, considering the $q+1$ hyperplanes through $L$ in $\mathrm{PG}(3, q)$ we conclude that $q$ have multiplicity $q$ and one has multiplicity $q(q-1)$, where the latter contradicts Corollary 34 .

We remark that the preceding result can be used to simplify the proof of the cylinder conjecture for $q=5$, although it is not necessary.

Lemma 44. For a set of $q(q-3)$ points in $\mathrm{PG}(2, q), q \geq 7$, there exists a line with a multiplicity not in $\{0, q-3, q-2\}$.

PROOF. Otherwise the first two standard equations would give $a_{q-2}=a_{0}(q-3)-(q-3)$ and $a_{q-3}=$ $q^{2}+2 q-2-a_{0}(q-2)$ so that the third yields

$$
0=a_{q-3}\left(\begin{array}{c}
q-3 \\
2
\end{array}\right)+a_{q-2}\left(\begin{array}{c}
q-2 \\
2
\end{array}\right)-\left(\begin{array}{c}
q(q-3) \\
2
\end{array}\right)=\frac{(q-3)\left(a_{0}(q-2)-4 q+2\right)}{2}
$$


which implies

$$
a_{0}=\frac{4 q-2}{q-2}=4+\frac{6}{q-2}
$$

Since $a_{0} \in \mathbb{N}_{0}$, we have $q \in\{3,4,5,8\}$. Due to our assumption $q \geq 7$ it remains to exclude the case $q=8$, where $a_{0}=5, a_{5}=48$, and $a_{6}=20$. Consider a 6 -line $L$. The only possibility for the distribution of the multiplicities of the lines through a 0 -point on $L$ is given by $0^{2} 5^{2} 6^{5}$, so that there are $3 \cdot 2=60$-lines. This contradicts $a_{0}=5$.

The assumption $q \geq 7$ in Lemma 44 is necessary, see Remark 42 for a counterexample for $q=5$ and the generator matrix

for $q=4$.

$$
\left(\begin{array}{llll}
1 & 1 & 0 & 0 \\
1 & 0 & 1 & 0 \\
1 & 0 & 0 & 1
\end{array}\right)
$$

Corollary 45. Under $(\star)$, there can be no $q(q-3)$-plane.

Proof. Due to Corollary 35, Corollary 40, and Proposition 41 we can assume $q \geq 7$. Lemma 43 implies that the maximum line multiplicity is at most $q-2$. If $H$ is a $q(q-3)$-plane, then Lemma 32 and Lemma 44 yield a contradiction.

Lemma 46. For a set of $q(q-4)$ points in $\mathrm{PG}(2, q), q \geq 7$, with line multiplicities contained in $\{0, q-2, q-$ $3, q-4\}$, we need $q=7$ and spectrum $\left(a_{0}, a_{q-4}, a_{q-3}, a_{q-2}\right)=(8,28,21,0)$.

PROOF. Solving the standard equations gives

so that $a_{q-2} \geq 0$ implies

$$
\begin{aligned}
& a_{q-2}=\frac{(q-4)(5 q-3)}{2}-\frac{(q-3)(q-4)}{2} a_{0} \\
& a_{q-3}=-(q-4)(5 q-2)+(q-2)(q-4) a_{0} \\
& a_{q-4}=\frac{7 q^{2}-19 q+6}{2}-\frac{(q-2)(q-3)}{2} a_{0},
\end{aligned}
$$

$$
a_{0} \leq \frac{5 q-3}{q-3}=6-\frac{q-15}{q-3}
$$

and $a_{q-3} \geq 0$ implies

$$
a_{0} \geq \frac{5 q-2}{q-2}=5+\frac{8}{q-2}
$$

So, for $q \geq 16$ we have $a_{0} \notin \mathbb{N}_{0}$, which is a contradiction.

For $7 \leq q \leq 13$ we can see that $a_{q-3}>0$. So consider a $(q-3)$-line $L$. Through each of the four 0 -points on $L$ there go at least two 0 -lines, since otherwise $1 \cdot 0+(q-1) \cdot(q-4)+1 \cdot(q-3)=q(q-4)+1>|\mathcal{K}|$. Therefore, we have $a_{0} \geq 8$, which is only possible for $q=7$. The spectrum then follows from the equations above.

Lemma 47. Under $(\star)$ for $q=7$, the spectrum is given by $a_{0}=22, a_{7}=357, a_{14}=21$ and $a_{21}=0$.

Proof. First we will show that $a_{21}=0$. Assume to the contrary the existence of a 21-plane $H$. From Lemma 46 we conclude that the spectrum $\left(b_{i}\right)$ of $\mathcal{S} \cap H$ satisfies $b_{0}=8, b_{3}=28, b_{4}=21$, and $b_{i}=0$ otherwise. Consider the possible hyperplane distributions through a 0 -line in $H: 0^{5} 7^{1} 21^{2}, 0^{5} 14^{2} 21^{1}, 0^{4} 7^{2} 14^{1} 21^{1}$, and $0^{3} 7^{4} 21^{1}$. In each case there are at least three 0 -planes through a 0 -line in $H$, so that there are at least $3 b_{0}=240$-planes in total. However, solving the standard equations for $\left\{a_{0}, a_{7}, a_{14}\right\}$ gives $a_{0}=22-a_{21}$, $a_{7}=357+3 a_{21}$, and $a_{14}=21-3 a_{21}$, so that $a_{0} \leq 22$, a contradiction. Therefore $a_{21}=0$ and the values for $a_{0}, a_{7}$ and $a_{14}$ follow immediately as well.

Remark 48. After 1671 seconds of computation time, QextNewEdition claims that there no sets of 14 points with line multiplicity at most 5 in $\operatorname{PG}(2,7)$ without lines of multiplicity 1 . If we additionally assume that there is no line of multiplicity 5 , then 908 seconds of computation time are needed. Using Lemma 47 this implies the truth of the cylinder conjecture for $(4,1,7)$. 
In the following we want to give an alternative, computer-free proof of the cylinder conjecture for $(4,1,7)$.

Lemma 49. A set $\mathcal{K}$ of 14 points in $\mathrm{PG}(2,7)$, whose line multiplicities are contained in $\{0,2,3,4,5\}$ has spectrum $\left(a_{0}, a_{2}, a_{3}, a_{4}, a_{5}\right)$ either $(10,37,2,8,0)$, (11,31, 10,5,0), (12, 25, 18, 2, 0), (11,30,13,2,1), or $(10,36,5,5,1)$.

PROOF. Solving the standard equations for $\left\{a_{0}, a_{2}, a_{3}\right\}$ gives

$$
\begin{aligned}
& a_{0}=\frac{38}{3}-a_{5}-\frac{1}{3} a_{4} \\
& a_{2}=21+5 a_{5}+2 a_{4} \\
& a_{3}=\frac{70}{3}-5 a_{5}-\frac{8}{3} a_{4}
\end{aligned}
$$

From $a_{0} \in \mathbb{N}_{0}$ we conclude $a_{4} \equiv 2(\bmod 3)$, so that especially $a_{4} \geq 2$. With this, $a_{3} \in \mathbb{N}_{0}$ yields $a_{4} \leq 8$ and $a_{5} \leq 3$.

In order to show $a_{5} \leq 1$ we consider a 5 -line $L$. Now, let $P$ be an arbitrary 1-point on $L$ and $Q$ be an arbitrary 0-point on $L$. Counting the points on the lines $L, L_{1}, \ldots, L_{7}$ through $P$ gives

$14=|\mathcal{K}|=\mathcal{K}(L)+\sum_{i=1}^{7} \mathcal{K}\left(L_{i}\right)-7 \cdot \mathcal{K}(P)=\mathcal{K}\left(L_{1}\right)+\sum_{i=2}^{7} \mathcal{K}\left(L_{i}\right)-2 \geq \mathcal{K}\left(L_{1}\right)+6 \cdot 2-2=\mathcal{K}\left(L_{1}\right)+10$

so that there is no 5 -line through $P$ besides $L$. Now assume that $M$ is a 5 -line through $Q$ and let $R$ be a 0 -point on $M$ not equal to $Q$. By $L_{0}^{\prime}, \ldots, L_{7}^{\prime}$ we denote the lines through $R$, where we assume $L_{0}^{\prime}=M$. Since five of the lines $L_{1}^{\prime}, \ldots, L_{7}^{\prime}$ hit $L$ in a point they have multiplicity at least 2 , which yields at least $5+5 \cdot 2=15>14$ points in $\mathcal{K}$, which is a contradiction. Thus, there is also no 5 -line through $Q$ besides $L$, so that $a_{5}$ is at most 1 .

To sum up, if $a_{5}=0$, then $a_{3} \in \mathbb{N}_{0}$ implies $a_{4} \in\{2,5,8\}$, and if $a_{5}=1$, the $a_{3} \in \mathbb{N}_{0}$ implies $a_{4} \in\{2,5\}$. Plugging this into the above equations gives the five stated spectra.

Note that we have applied the same "technique" to conclude $a_{5} \leq 1$ as the one used in the proof of Lemma 43 . We will now rule out all possibilities from Lemma 49.

Lemma 50. A set $\mathcal{K}$ of 14 points in $\mathrm{PG}(2,7)$, whose line multiplicities are contained in $\{0,2,3,4,5\}$, can not have 5-lines.

Proof. Let $L$ be a 5 -line and $Q_{1}, Q_{2}$, and $Q_{3}$ be the 0 -points on $L$. Define a new set $\mathcal{K}^{\prime}$ as the symmetric difference of $\mathcal{K}$ and $L: \mathcal{K}^{\prime}=\mathcal{K} \backslash(L \cap \mathcal{K}) \cup\left\{Q_{1}, Q_{2}, Q_{3}\right\}$. As $\mathcal{K}$ did not have any 1 -lines, we can see that $\mathcal{K}^{\prime}$ is a non-trivial blocking set of size 12 in $\mathrm{PG}(2,7)$. Blocking sets of cardinality 12 in $\mathrm{PG}(2,7)$ not containing a line have been classified in [2]: besides the projective triangle there exists a unique sporadic example of non-Rédei type. Both of these constructions share the property that there exists at least one 2-line through every 1-point. Now considering the point $Q_{1}$, we can easily see that there are no 2 -lines to $\mathcal{K}^{\prime}$ through it, as they would have to come from 1 -lines to $\mathcal{K}$, which do not exist.

Lemma 51. No set $\mathcal{K}$ of 14 points in $\mathrm{PG}(2,7)$ with spectrum $\left(a_{0}, a_{2}, a_{3}, a_{4}\right)=(11,31,10,5)$ and $a_{i}=0$ otherwise exists.

Proof. The three possible distributions of the multiplicities of the lines through a 1-point are $4^{2} 3^{1} 2^{5}, 4^{1} 3^{3} 2^{4}$, $3^{5} 2^{3}$ and we speak of type $A_{1}, A_{2}$, and $A_{3}$, respectively.

Assume that $P$ is a 1-point of type $A_{3}$ and let $L$ be an arbitrary 3-line through $P$. The five 0 -points on $L$ are contained in another 3 -line besides $L$, by parity considerations. Thus by $a_{3}=10$, the two 1 -points on $L$ that are not equal to $P$ are of type $A_{1}$. Since $L$ was chosen arbitrary, all ten 1-points on 3-lines through $P$ that are not equal to $P$ are of type $A_{1}$. Let $Q_{1}, Q_{2}$, and $Q_{3}$ denote the three other 1-points not equal to $P$ and not forming a 3-line with $P$. All of the five 3-lines not incident with $P$ have to consist of 1-points in $\left\{Q_{1}, Q_{2}, Q_{3}\right\}$, which is impossible. Thus, there is no 1-point of type $A_{3}$.

Consider an arbitrary 3-line $L^{\prime}$. The five 0-points on $L^{\prime}$ are contained in another 3-line besides $L^{\prime}$, for the same reason as before. Thus not all three 1-points on $L^{\prime}$ can be of type $A_{2}$ as $a_{3}=10$. So each 3-line contains at least one 1-point of type $A_{1}$, which is then contained in no other 3 -line. This means that there are at least ten 1 -points of type $A_{1}$. Counting the 4 -lines, this gives $a_{4} \geq(10 \cdot 2+4 \cdot 1) / 4=6>5$, which is a contradiction. 
Proposition 52. The generalized cylinder conjecture is true for the triple $(4,1,7)$.

Proof. Assume the existence of a counterexample $\mathcal{S}$. Due to Lemma 47, there exists a hyperplane $H$ so that $\mathcal{S} \cap H$ is a set of 14 point whose line multiplicities are contained in $\{0,2,3,4,5\}$. From Lemma 49, Lemma 50 and Lemma 51 the spectrum of $\mathcal{S} \cap H$ is given by $\left(b_{0}, b_{2}, b_{3}, b_{4}, b_{5}\right)=(12,25,18,2,0)$. Note that every $j$-line in $H$, where $j \geq 2$, is contained in $(j-1)$ planes of multiplicity 14 and $(9-j)$ planes of multiplicity 7 in $\mathrm{PG}(3,7)$. Thus $\mathcal{S}$ contains at least $b_{3}+2 b_{4}=18+2 \cdot 2=22$ planes of multiplicity 14 , which contradicts $a_{14}=21$.

While our computer-free proof of the cylinder conjecture for $(4,1,7)$ is rather lengthy, most parts are more or less systematic and might be generalized to larger field sizes. A big obstacle is that we cannot prove the truth of the observation in Remark 48 directly. Actually, we do not have a complete proof of this specific nonexistence result sets of 14 points in $\operatorname{PG}(2,7)$ without 1-lines and just sailed around the remaining open case in the proof of Proposition 52. Maybe other methods are more suitable for this kind of problems in $\mathrm{PG}(2, q)$. Of course, allowing computer enumerations drastically reduces the length of the argumentation. Starting from Lemma 32 and Lemma 32 we can computationally exclude many possibilities for the restriction $\mathcal{S} \cap H$ for a hyperplane $H$. In other words, the possible multiplicities for the weights $\mathcal{S}(H)$ for the hyperplanes can be restricted by enumeration results for 3 -dimensional codes over $\mathbb{F}_{q}$. By considering a subcode of the 4dimensional projective code corresponding to $\mathcal{S}$ we obtain a $q^{2}$-divisible $\left[q^{2}-1,3\right]_{q}$-code $C$ with a restricted set of weights that might also be enumerated computationally. For our example we remark that there are 54 non-isomorphic $[48,3,\{21,28,35,42\}]_{7}$-codes and 46 non-isomorphic $[48,3,\{28,35,42\}]_{7}$-codes. Moreover, the information that $\mathcal{S}$ does not contain a full affine line restricts the possible residual codes of codewords in $C$. By that criterion 6 of of the 46 non-isomorphic $[48,3,\{28,35,42\}]_{7}$-codes can be excluded. Similar restrictions can arise from the previously mentioned classification of projective $[k q, 3,\{(k-1) q+1, \ldots, k(q-$ 1 ), $k q\}]_{q}$-codes. E.g., as also theoretically proven, all projective $[21,3,\{15,16,17,18,21\}] 7$-codes do not contain codewords of weight 15 or 16 . So, in the residual code of a codeword of weight 28 in $C$ the weights 15 and 16 cannot occur. This excludes 12 further codes. For the remaining twenty-eight $[48,3,\{28,35,42\}]_{7^{-}}$ codes we can computationally check whether an extension to a projective $[49,4,\{28,35,42\}]_{7}$-code exists. To this end we can utilize and ILP formulation and an ILP solver. We remark that the tightest ILP instance needed 1238996 branch\&bound nodes and 28.75 hours of computation time. At the very least, this approach gives a computational verification of Proposition 52.

Let us finish with some conclusions for the generalized cylinder conjecture for $q=8$. Assume that $\mathcal{S}$ is an 8-divisible spanning set of 64 points in $\mathrm{PG}(3, q)$ that is not a cylinder. From Corollary 34, Corollary 39, Corollary 45 and Lemma 46, we conclude that the hyperplane multiplicities with respect to $\mathcal{S}$ are contained in $\{0,8,16,24\}$.

Solving the standard equations for the spectrum $\left(a_{i}\right)$ of $\mathcal{S}$ gives

$$
\begin{aligned}
a_{0} & =29-a_{24} \\
a_{8} & =528+3 a_{24} \\
a_{16} & =28-3 a_{24},
\end{aligned}
$$

so that $a_{0} \leq 29$. Now assume that $H$ is a 24-plane and consider the spectrum $\left(b_{i}\right)$ of $\mathcal{S} \cap H$. Solving the standard equations for $\left\{b_{3}, b_{5}, b_{6}\right\}$ gives

$$
\begin{aligned}
& b_{3}=97-5 b_{0}-\frac{b_{4}}{3} \\
& b_{5}=-69+9 b_{0}-b_{4} \\
& b_{6}=45-5 b_{0}+\frac{b_{4}}{3},
\end{aligned}
$$

so that $b_{5} \geq 0$ implies $b_{0} \geq\left\lceil\frac{69}{9}\right\rceil=8$. Since through every 0 -line in $H$ there are at least three 0 -planes, $a_{0} \leq 29$ implies $b_{0} \leq\left\lfloor\frac{29}{3}\right\rfloor=9$. For $b_{0}=8$ we have $b_{3}=62-b_{6}, b_{4}=-15+3 b_{6}$, and $b_{5}=18-3 b_{6}$, so 
that either

$$
\left(b_{0}, b_{3}, b_{4}, b_{5}, b_{6}\right) \in\{(8,57,0,3,5),(8,56,3,0,6)\}
$$

or

$$
\left(b_{0}, b_{3}, b_{4}, b_{5}, b_{6}\right)=\left(9,52-b_{6}, 3 b_{6}, 12-3 b_{6}, b_{6}\right),
$$

where $0 \leq b_{6} \leq 4$. Consider a 4 -line $L$. Through each of the five 0 -points on $L$ there are at least two incident 0 -lines, so that $b_{0} \geq 5 \cdot 2=10$. Thus, we conclude

$$
\left(b_{0}, b_{3}, b_{4}, b_{5}, b_{6}\right) \in\{(8,57,0,3,5),(9,52,0,12,0)\} .
$$

For the second case consider a 1-point $P$. Since all lines through $P$ have to be 3 - or 5 -lines, we have $|\mathcal{S} \cap H| \equiv$ $1(\bmod 2)$, which is a contradiction. For the first case we consider a 5 -line $L$ and observe that the unique possibility for the distribution of the multiplicities of the lines through a 1-point on $L$ is given by $3^{7} 5^{1} 6^{1}$. Thus, besides $L$, there remain eight 0 -lines, twenty-two 3 -lines, and two 5 -lines for the four 0 -points on $L$. The only possibility for a 0 -point, using only 0 -, 3 -, and 5 -lines, is $0^{3} 3^{3} 5^{3}$ for the distribution of the multiplicities of the incident lines. This case cannot occur four times, so that we finally conclude $a_{24}=0$, which leaves the unique spectrum $\left(a_{0}, a_{8}, a_{16}\right)=(29,528,28)$ for $\mathcal{S}$. The above considerations are elementary and easy, but a bit ad hoc. As for $q=7$, we are again in a situation where it seems that we are missing the right tools to tackle the problem in an elegant way. Of course, it is very likely that the cylinder conjecture is true for $q=8$.

\section{REFERENCES}

[1] S. Ball. On the graph of a function in many variables over a finite field. Des. Codes Cryptogr., 47(1-3):159-164, 2008.

[2] A. Blokhuis, A. E. Brouwer, and H. A. Wilbrink. Blocking sets in $\mathrm{PG}(2, p)$ for small $p$, and partial spreads in $\mathrm{PG}(3,7)$. Adv. Geom., (suppl.):S245-S253, 2003. Special issue dedicated to Adriano Barlotti.

[3] J. De Beule, J. Demeyer, S. Mattheus, and P. Sziklai. On the cylinder conjecture. Des. Codes Cryptogr., 87(4):879-893, 2019.

[4] P. Govaerts and L. Storme. On a particular class of minihypers and its applications. I. The result for general q. Des. Codes Cryptogr., 28(1):51-63, 2003.

[5] T. Honold, M. Kiermaier, and S. Kurz. Partial spreads and vector space partitions. In Network Coding and Subspace Designs, pages 131-170. Springer, 2018.

[6] S. Kurz. Lincode - computer classification of linear codes. arXiv preprint 1912.09357, 2019.

[7] M. Lavrauw and G. Van de Voorde. Field reduction and linear sets in finite geometry. In Topics in finite fields, volume 632 of Contemp. Math., pages 271-293. Amer. Math. Soc., Providence, RI, 2015.

[8] L. Lovász and A. Schrijver. Remarks on a theorem of Rédei. Studia Sci. Math. Hungar., 16(3-4):449-454, 1983.

[9] L. Rédei. Lückenhafte Polynome über endlichen Körpern. Birkhäuser Verlag, Basel-Stuttgart, 1970.

[10] G. Van de Voorde. On sets without tangents and exterior sets of a conic. Discrete Math., 311(20):2253-2258, 2011.

[11] H. N. Ward. Divisible codes—a survey. Serdica Math. J., 27(4):263-278, 2001

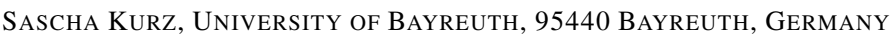

Sam Mattheus, Universiteit Brussel, 1050 Elsene, Belgium

Email address: sascha.kurzeuni-bayreuth.de; sam.mattheusevub.ac.be 